\title{
Report of the Annual Meeting
}

Rapports annuels de la Société historique du Canada

\section{Membership of the Canadian Historical Association}

Volume 13, numéro 1, 1934

URI : https://id.erudit.org/iderudit/300139ar

DOI : https://doi.org/10.7202/300139ar

Aller au sommaire du numéro

\section{Éditeur(s)}

The Canadian Historical Association/La Société historique du Canada

\section{ISSN}

0317-0594 (imprimé)

1712-9095 (numérique)

Découvrir la revue

Citer ce document

(1934). Membership of the Canadian Historical Association. Report of the Annual Meeting / Rapports annuels de la Société historique du Canada, 13(1), 129-135. https://doi.org/10.7202/300139ar d'utilisation que vous pouvez consulter en ligne. 


\title{
MEMBERSHIP OF THE CANADIAN HISTORICAL ASSOCIATION
}

\author{
AFFILIATED SOCIETIES AND ORGANIZATIONS
}

Acadia University Library, Wolfville, N.S.

Antiquarian and Numismatic Society of Montreal, Chateau de Ramezay. Victor Morin, LL.D., President, 57 rue Saint-Jacques Ouest, Montreal, P.Q.; Pemberton Smith, Treasurer, 414 St. James St., Montreal, P.Q.

Antiquarian Society of Montreal (Women's Branch). Madame J. I. Tarte, President; Miss Lois C. Malcolm, Secretary, 1 Rosemount Ave., Apt. 63, Westmount, P.Q.

British Columbia Historical Association. F. V. Longstaff, President, Victoria, B.C. G. M. McTavish, Hon. Treasurer, Victoria, B.C.; H. T. Nation, Hon. Secretary, 2380 Windsor Road, Victoria, B.C.

Canadian Military Institute, 426 University Ave., Toronto, Ont.

Clark University Library, Worcester, Mass., U.S.A. Louis N. Wilson, Librarian.

Cleveland Public Library, 325 Superior Ave., N.E., Cleveland, Ohio, U.S.A. Miss Leta E. Adams, Order Librarian.

Hamilton Public Library. Mrs. Lurene Lyle, Librarian, Hamilton, Ont.

Henry E. Huntington Library and Art Gallery, San Marino, Cal. Leslie E. Bliss, Librarian.

Hislorical Society of Alberta. Dr. A. C. Rutherford, President; W. Everard Edmonds, Secretary, 1146-91st Ave., Edmonton, Alta.; M. H. Long, Treasurer.

Kingston Historical Society. H. F. Price, President; W. S. Lavell, Secretary, 151 Earl St., Kingston, Ont.; J. H. Mitchell, Treasurer, 87 L. Union St., Kingston, Ont.

Last Post Fund. Arthur H. D. Hair, Secretary-treasurer, P.O. Box 1382, Montreal, P.Q.

Legislative Library of Ontario. A. F. Wilgress, Legislative Librarian, Toronto, Ont.

Library of Parliament. Felix Desrochers, General Librarian; Hon. Martin Burrell, Parliamentary Librarian, Ottawa, Ont.

Literary and Historical Society of Quebec. Count de Bury, President; A. Robertson, Recording Secretary; Col. F. M. Stanton, Corresponding Secretary; R. L. Smyth, Treasurer, P.O. Box 187, Quebec, P.Q.

London and Middlesex Historical Society. Rev. M. A. Garland, President, Ilderton, Ont.; Fred Landon, Treasurer, University of Western Ontario, London, Ont.

London Public Library. Richard E. Crouch, Librarian, London, Ont.; James S. Bell, Treasurer.

McGill University Library. Gerhard R. Lomer, M.A., Ph.D., Librarian, Montreal, P.Q.

Niagara Historical Society. Mrs. C. E. Brown, Treasurer, Niagara-on-the-Lake, Ont. Norwich Pioneers' Society. A. L. Bushell, Secretary, Norwich, Ont.

Nova Scolia Historical Society. B. E. Paterson, President; Mrs. G. A. McIntosh, Corresponding Secretary; W. L. Payzant, Recording Secretary; 1. L. Wainwright, Treasurer, P.O. Box 370, Halifax, N.S.

Ohio State University, University Library, Columbus, Ohio, U.S.A.

Ontario Historical Society. J. McE. Murray, Secretary-treasurer, Normal School Building, Toronto, Ont.

Point Grey Junior High School, 37th Ave. and E. Boulevard, Vancouver, B.C. H. N. MacCorkindale, Principal.

Provincial Library of Alberia. Colin G. Groff, Acting Librarian, Edmonton, Alta.

Provincial Library of British Columbia. Dr. W. Kaye Lamb, Provincial Librarian and Archivist, Parliament Buildings, Victoria, B.C.

Provincial Library of Manitoba. W. J. Healy, Provincial Librarian, Winnipeg, Man.

Thunder Bay Historical Society. Miss M. J. L. Black, President; Mrs. E. R. Patrick, Secretary-treasurer, 54 Shuniah Rd., Port Arthur, Ont.

Toronto Public Library. George H. Locke, M.A., LL.D., Librarian, College St., Toronto, Ont.

University of California Library, Berkeley, Cal., U.S.A. Harold L. Leupp, Librarian. University of Cincinnati Library, Burnet Woods Park, Cincinnati, Ohio, U.S.A.

University of Manitoba Library. Frank E. Nuttall, Librarian, Fort Garry Site, Winnipeg, Man. 
University of Toronto Library. W. S. Wallace, Librarian, Toronto, Ont.

University of Western Ontario, University Library, London, Ont.

Women's Canadian Historical Society of Ottawa. Mrs. J. Lorne McDougall, President, 560 Parkdale Ave., Ottawa, Ont.; Mrs. H. K. Carruthers, Recording Secretary, 57 1st Ave., Ottawa, Ont.; Mrs. James Dick, Corresponding Secretary, 401 Albert St., Ottawa, Ont.; Mrs. B. L. York, Treasurer, 112 Strathcona Ave., Ottawa, Ont. Women's Canadian Historical Society of Toronto. Miss C. Roberts, President, 20 Earl St., Toronto, Ont.; Mrs. Seymour Corley, Recording Secretary; Mrs. F. M. Fairbrother, Corresponding Secretary; Mrs. W. A. Parks, Treasurer, 69 Albany Ave., Toronto, Ont.

Women's Wentworth Historical Society. Mrs. Lynch-Staunton, President; Mrs. Bertie D. Smith, Secretary, 155 Hughson St. S., Hamilton, Ont.; Mrs. W. H. Magill, Treasurer.

\section{(B) LIFE MEMBERS}

Bacon, N. H., Hudson's Bay Company, 17 St. John St., Montreal

Brown, Sir Geo. McL., European General Manager, C.P.R., Trafalgar Square London, England

Curry, Hon. N., 581 Sherbrooke St. W., Montreal

Dow, Miss Jessie, Ritz Carlton Hotel, Montreal

Gosselin, L. A., 501 St. Catherine Rd., Outremont, P.Q.

Hardy, Senator A. C., Brockville, Ont.

Hardy, Mrs. A. C., Brockville, Ont.

Hastings, G. V., 55 Donald St., Winnipeg

Holt, Sir Herbert, 3459 Stanley St., Montreal

Kindersley, Sir R., Langley House, Abbots Langley, Herts, England

Laurie, W. Pitt, 202 St. Louis Rd., Quebec

Lyman, A. C., 3520 McTavish St., Montreal
Macfarlane, Miss J. J., 527 Sherbrooke St. W., Montreal

Masters, C. H., 220 Bay St., Ottawa

Mills, Col. Dudley, 24 Washington House, Basil St., London, S.W. 3, England

Musson, Chas. J., 17 Wilton Ave., Toronto

Raymond, Hon. Donat, 3541 Ontario St., Montreal

Riordan, Carl, 374 Côte des Neiges Road, Montreal

Ross, Com. J. K. L., 107 St. James St., Montreal

Simpson, Mrs. J. B., 173 Percy St., Ottawa

Smith, Pemberton, 414 St. James St. W., Montreal

Vaughan, H. H., Dominion Bridge Co., Ltd., Lachine, P.Q.

Wilson, Hon. Cairine, Rockcliffe, Ont.

\section{(C) ANNUAL MEMBERS}

Adair, E. R., McGill University, Montreal Alberts, Gerald A., 17 Fanshaw Ave., Yonkers, N.Y.

Allard, Rev. Father J. A., East Bathurst, Gloucester Co., N.B.

Allard, Hon. Jules, Palais de Justice, Montréal

Anderson, Dr. H. B., 338 Bloor St. E., Toronto

Angers, M. Philippe, Régistrateur, Division d'Enregistrement du Comté de Beauce, Beauceville Est, P.Q.

Atherton, Dr. W. H., 329 Common St., Montreal

Audet, Francis J., Public Archives of Canada, Ottawa

Baldwin, Robert McQueen, 35 Heath St. W., Toronto

Barbeau, C. M., 260 McLaren St., Ottawa

Barber, Lieut.-Col. R. R., c/o Kilmer Irving and Davis, 10 Adelaide St. E., Toronto

Bate, W. T., Box 302, St. Catharines, Ont.

Baylis, S. M., 3574 University St., Montreal
Bédard, M. l'abbé Lucien, 14 Dolbeau St., Québee

Bell, Dr. C. N., 121 Carleton St., Winnipeg Bernier, Capt. J. E., 27 Fraser St., Lévis, P.Q.

Bertrand, J. P., 306 Scott Block, Winnipeg

Birks, W. M., 254 Stanley St., Montreal

Bissett, Alex., P.O. Box 1390, Montreal

Bonham, Milledge L., Jr., Hamilton College, Clinton, N.Y.

Boothroyd, E. E., Bishop's College, Lennoxville, P.Q.

Borden, Sir Robert, 201 Wurtemburg St., Ottawa

Brady, Alexander, Economics Building, University of Toronto, Toronto

Brand, Dr. C. F., Dept. of History, Stanford University, Cal.

Brebner, J. Bartlet, Dept. of History, Columbia University, New York, N.Y.

Brisebois, Napoléon, 1931 rue Centre, Montréal

British Museum, Dept. of Printed Books, London, W.C. 1, England 


\section{(C) ANNUAL MEMBERS-Continued}

Brown, A. T., c/o E. Pullan, Ltd., Toronto Brown, George W., Baldwin House, University of Toronto, Toronto

Brown, Miss Vera Lee, Hatfield House, Smith College, Northampton, Mass.

Browne, Lieut.-Col. Peter L., 3766 Côte des Neiges Road, Montreal

Bruce, Hon. R. Randolph, Invermere, B.C.

Buchanan, A. W. P., Dominion Express Building, 190 St. James St., Montreal

Burpee, L. J., 22 Rideau Terrace, Ottawa

Burt, A. L., University of Minnesota, Minneapolis, Minn.

Butchart, R. P., Tod Inlet, Vancouver Island, B.C.

Byers, Mrs. R. P., 43 Walmsley Blvd., Toronto

Cantlie, Lieut.-Col. G. S., 1106 Sherbrooke St. W. Montreal

Cardin, Hon. P. J. Arthur, House of Commons, Ottawa

Caron, M. l'abbé Ivanhoë, 10 rue Crémazie, Québec

Caty, Charles, 1540 Bernard Ave. W., Apt. 36, Outremont, P.Q.

Cayley, Chas. E., Trinity University, Waxahachie, Texas

Chapais, Hon. Thomas, The Senate, Ottawa

Chartier, Chanoine, Université de Montréal, 1265 rue St.-Denis, Montréal

Chicoutimi, Séminaire de, Chicoutimi, P.Q

Chrysler, F. H., K.C., 41 Central Chambers, Ottawa

Clark, John C, 42 Playter Crescent, Toronto

Clay, Charles, 607 Langside St., Winnipeg

Clergue, F. H., Suite 1815-17, 360 St. James St., Montreal

Coats, R. H., 176 Manor Ave., Rockcliffe, Ont.

Cochrane, Chas. N., Dean's House, University College, University of Toronto, Toronto

Cockshutt, W. F., Brantford, Ont.

Colby, C. W., 1240 Pine Ave. W., Montreal

Connell, Allison B., K.C., Woodstock, N.B.

Cooke, A. C., University of British Columbia, Vancouver

Corless, C. V., Mond Nickel Co., Tillsonburg, Ont.

Coutts, G. B., 517 Seventh Ave. W., Calgary

Coyne, Dr. James H., St. Thomas, Ont.

Crane, J. W., Medical School, University of Western Ontario, London, Ont.

Creighton, Donald G., Baldwin House, University of Toronto, Toronto

Creighton, J. H., 3539 Welwyn St., Vancouver
Cross, Jesse E., 99 Joralemon St., Brooklyn, N.Y.

Crouse, Nellis M., 414 Cayuga Heights Road, Ithaca, N.Y.

Crowe, Judge 'W., Judges' Chambers, County Court, Sydney, N.S.

Cunningham, Lieut.-Col. J. F., 400 Laurier Ave. E., Ottawa

Dadson, Thomas, Wolfville, N.S.

Dandurand, Hon. Sénateur R., 548 rue Sherbrooke-ouest, Montréal

Davidson, Jas., 739 Mountain St. Montreal

Delage, Hon. Cyrille F., Surintendant Instruction Publique, Palais législatif, Québec

Demers, M. le juge Philippe, Palais de Justice, Montréal

Desaulniers, Gonzalve, Palais de Justice. Montréal

Desjardins, C. A. R., St. André de Kamouraska, P.Q.

Desranleau, P. S., Chanoine, Presbytère de St-Pierre, Sorel, P.Q.

Desrosiers, M. l'abbé Adélard, Principal de l'École Normale, Montréal

Dewey, A. Gordon, Dept. of Political Science, Union College, Schenectady, N.Y.

Dobbin, R. L., 622 George St., Peterborough, Ont.

Donald, Dr. J. T., 1181 Guy St., Montreal

Dorland, Arthur Garratt, University of Western Ontario, London, Ont.

Doughty, Dr, A. G., Public Archives of Canada, Ottawa

Drummond, Lady, 3432 Drummond St., Montreal

Drysdale, Wm. F., 418 Pine Ave. W., Montreal

Durnford, Miss M. G., 4216 Western Ave., Apt. 6, Westmount, P.Q.

Eastman, Mack, International Labour Office, Geneva, Switzerland

Edgar, Miss M. C., 507 Guy St., Montreal

Elliott, T. C., Box 775, Walla Walla, Washington

Ells, Miss Margaret, Public Archives of Nova Scotia, Halifax

Eschambault, Rev. Antoine d', Archéveché, St. Boniface, Man.

Fabre-Surveyer, Hon. Juge E., 128 Avenue Maplewood, Outremont, Montreal

Farlinger, Miss Isabella K., "Belmont", Morrisburg, Ont.

Fauteux, Aegidius, The Public Library of the City of Montreal, Montreal

Fee, Norman, Public Archives of Canada, Ottawa

Fieldhouse, H. N., Dept of History, University of Manitoba, Winnipeg

Finlayson, D. K., Principal, Inverness Schools, Inverness, N.S. 


\section{(C) ANNUAL MEMBERS-Continued}

Fisher, H. M., 1302 Melrose Ave. Saskatoon

Flavelle, Sir Jos. W., Bart., 25 King St. W., Toronto

Fleming, Roy F., 47 Roseberry Ave., Ottawa

Flenley, R., Baldwin House, University of Toronto, Toronto

Foran, T. P., K.C., 63 Main St., Hull, P.Q.

Forsey, Eugene, McGill University, Montreal

Fosbery, C. S., Lower Canada College, Montreal

Foss, Carl Alstien, 823 Pine Place, Bellmore, L.I., N.Y.

Foster, Miss Joan, Rothesay, N.B.

Fotheringham, Maj.-Gen. J. T., 20 Wellesley St., Toronto

Frigon, A. P., 157 rue Sherbrooke-est, Montreal

Frind, Herbert O., F.R.G.S., Ontario Club, Toronto, Ont.

Galipeault, Hon. Antonin, Palais de Justice, Québec.

Gareau, Rév. Père J. B., C.S.V., 1145 St.-Viateur, Outremont, P.Q.

Garland, Rev. M. A., Ilderton, Ont.

Garneau, Sir J. Georges, 136 Grande Allée Québec

Gates, Mrs. Paul W., 2434 Tunlaw Rd. N.W. Washington, D.C.

Geological Survey Library, Victoria Museum, Ottawa

Gérin, Léon, Coaticook, P.Q.

Glazebrook, G. de T., Baldwin House, University of Toronto, Toronto

Golden, Harvey, 265 Mount Royal Ave., Montreal

Gordon, Rev. C. W. (Ralph Connor), Winnipeg

Gordonsmith, C., Star Office, Montreal

Graham, Hon. Geo. P., Kenniston Apts., 345a Elgin St., Ottawa

Graham, Gerald S., Eliot House, O 11 , Cambridge, Mass.

Gravel, C. E., 545 ave. Davaar, Outremont, P.Q.

Green, Judge George Wellington, Court House, Medicine Hat, Alta.

Groulx, M. l'abbé Lionel, 847 rue Sherbrooke-est, Montréal

Guertin, Rev. L., C.S.C., St. Joseph University, St. Joseph, N.B.

Guilday, Rev. Peter, 305 Mullen Memorial Library, Catholic University of America, Washington, D.C.

Gunne, Dr. J. R., Dauphin, Man.

Hamilton, John, Union Bank Building, Quebec

Hamilton, Louis, Berlin-Lichterfelde-Ost. Lutherstr. 4A, Germany

Hanna, W. E., 182 Rosewell Ave., Toronto

Harkin, J. B., Commissioner of Dominion Lands, Dept. of the Interior, Ottawa
Harkness, John G., K.C., Cornwall, Ont. Harvey, Hon. Chief Justice, 10226 Connaught Drive, Edmonton

Harvey, Daniel C., Public Archives of Nova Scotia, Halifax

Harwood, C. A. de Lotbinière, Room 1006, 355 McGill St., Montreal

Hickson, J. W. A., 3428 Ontario Ave., Montreal

Hingston, Lady, 1000 Sherbrooke St. W., Montreal

Hitchins, Fred H., 503 English St., London, Ont.

Howard, Mrs. M. M., 27 Springhurst Ave., Toronto

Howay, His Honour Judge F. W., Law Courts, New Westminster, B.C.

Hudson, A. B., Barrister, Winnipeg

Innis, Harold A., Economics Building, University of Toronto, Toronto

Institute of Historical Research, University of London, London, England

Ironsides, P.Q., Collège St-Alexandre

Jackson, Gilbert, Economics Building, University of Toronto, Toronto

Jackson, Mrs. K. B., 362 Glengrove Ave. W., Toronto

James, Major R. H., 434 University Ave., Toronto

Jarvis, W. H. P., Canton, Ont.

Jefferys, Charles W., York Mills, Ont.

Jenkins, John, 666 Belmont Ave., Westmount, P.Q.

Johnston, Robert A. A, 112 Old Forest Hill Rd., Toronto

Jonasson, Jonas A., Box 312, Salem, Ore.

Jones, George M., 371 Bloor St. W., Toronto

Kelly, Rev. Edward, St. Clare's Rectory, 133 Westmount Ave., Toronto

Kennedy, Howard Angus, P.O. Box 1424, Montreal

Kennedy, Miss Mary L., Provincial Library, Winnipeg

Kennedy, W. P. M., Law Building, University of Toronto, Toronto

Kenney, Dr. J. F., Public Archives of Canada, Ottawa

King, Right Hon. W. L. Mackenzie, Ottawa

LaBruère, Montarville Boucher de, Château de Ramezay, Montréal

Laflamme, M. l'abbé 'Eug.-C., 16 rue Buade, Québec

Laidlaw, John B., 12 Wellington St. E., Toronto

Laird, David H., K.C., 400 Victory Building, Winnipeg

Lanctôt, Dr. Gustave, Public Archives of Canada, Ottawa

Landon, Fred, 846 Hellmuth Ave., London, Ont.

Lapierre, E. A., Case 1930, Sudbury, Ont. 


\section{(C) ANNUAL MEMBERS-Continued}

Latchford, Hon. F. R., Osgoode Hall, Toronto

Laureys, Henry, 535 ave. Viger, Montréal Leacock, Stephen, McGill University, Montreal

LeDuc, Thomas H., 703 Hartley Hall, Columbia University, N.Y.

Lefebvre, Olivier, 59 Notre-Dame-est, Montréal

Léonard, Mgr. J. K., Evêque de Rimouski, P.Q.

Leonard, Col. Ibbotson, 782 Wellington St., London, Ont.

Lett, R. C. W., Natural Resources Branch, Industrial Dept., C.N.R., Vancouver

Library of Congress, Washington, D.C.

Loening, Mrs. Sarah L., Southampton, Long Island, N.Y.

Long, Mrs. Ernest E., 102 Collier St. Barrie, Ont.

Long, Morden H., University of Alberta, Edmonton

Longley, R. S., Dept. of History, Acadia University, Wolfville, N.S.

Longstaff, Major Frederick Victor, Seabank, 50 Highland Drive, Victoria

Lounsbury, R. G., 8 Devon Way, Hastings on Hudson, N.Y.

Lower, A. R. M., Wesley College, Winnipeg

McArthur, Duncan C., Dept. of Education, Parliament Bldgs., Toronto

MacDermot, T. W. L., League of Nations Society, Ottawa

Macdonald, Norman, Dept. of History, McMaster University, Hamilton, Ont

Macfarlane, R. O., University of Manitoba, Winnipeg

McInnes, C. M., University of Bristol, Bristol, England

McInnes, Hector, 35 Bedford St. S. Halifax

MacKay, Douglas, Canadian Committee Office, Hudson's Bay Co., Winnipeg

Mackintosh, W. A., Queen's University, Kingston

MacLaren, C. H., 14 Metcalfe St., Ottawa

MacLean, Dr. James A., University of Manitoba, Winnipeg

McLennan, Francis, Loretteville, P.Q.

McLennan, F. D., Drawer 908, Cornwall, Ont.

McLennan, Hon. J. S., The Senate, Ottawa

Macleod, J. E. A., 323 Fourth Ave. W., Calgary

McLetchie, James K., 5452 Snowdon Ave., Montreal

McMahon, E., 315 Claremont Ave., Westmount, P.Q.

McNeill, John T.,Dept. of Church History, University of Chicago, Chicago, Ill.

Macpherson, J. E., P.O. Drawer 150, Montreal
McQueen, J. M., 57 Renfrew Ave., Ottawa

MacTier, A. D., 474 Sherbrooke St. W. Montreal

Magor, J. H., 524 Mount Pleasant Ave. Westmount, P.Q.

Magrath, C. A., 398 Wilbrod St., Ottawa

Martin, Chester, Baldwin House, University of Toronto, Toronto

Massey, Hon. Vincent, Port Hope, Ont.

Maurault, Olivier, 1000 boul. Crémazieest, Montréal

Michaud, M. l'abbé Jos. D., Val-Brillant, Matane, P.Q.

Milner, Frank L., K.C., P.O. Box 1213 Halifax

Mitchell, Homer A., 487 Victoria Ave., Westmount, P.Q.

Moehlman, Arthur H., Barton Hills, Ann Arbor, Mich.

Molson, J. Dinham, c/o Bank of Montreal, Westmount, P.Q.

Montréal, Collège de, 1931 rue Sherbrooke-ouest, Montréal

Montréal, Collège Ste-Marie, 1180 rue Bleury, Montréal

Montréal, Commission des Écoles Catholiques

Moore, Miss Elsie, 2A The Locarno, 1 Roslyn Road, Winnipeg

Morden, W. S., K.C., 34 King St. E., Toronto

Morgan, F. Cleveland, 308 Peel St., Montreal

Morin, Victor, 57 rue Saint-Jacques ouest, Montréa!

Morton, Arthur S., University of Saskatchewan, Saskatoon

Mott, Miss K. Stella, R.R. No. 3, Norwich, Ont.

Muir, Miss M. Dudley, 1550 Mackay St., Apt. 4, Montreal

Mulligan, Rev. Wm. Orr, 351 Melville Ave., Westmount, P.Q.

Mulock, Sir William, Osgoode Hall Toronto

Munro, W. B., 268 Bellefontaine St. Pasadena, Cal.

Munroe, David, Lower Canada College, Montreal

Murphy, Miss Ethel, 128 Park St. Moncton, N.B.

Murray, W. H., 36 Churchill Ave., Westmount, P.Q.

Nadeau, Dr. Gabriel, Rutland State Sanatorium, Rutland, Mass.

Naftel, Frederick J., Goderich, Ont.

Neilly, Mrs. Balmer, 39 Woodlawn Ave., Toronto

Nelson, H. C., 346 Main St. W., North Bay, Ont.

New, Chester W., McMaster University, Hamilton, Ont.

O'Brien, A. H., 31 Glengowan Road, Toronto 


\section{(C) ANNUAL MEMBERS-Continued}

Oliver, Mrs. Frank, Edmonton

Orchard, Rev. F. Graham, England

Panabaker, D. N., Walker St., Hespeler, Ont.

Papineau, Mgr., Evêché de Joliette, Joliette, P.Q.

Parizeau, Gérard, 4152 ave. Marcil, Montréal

Paton, Hugh, 38 Victoria Sq., Montreal

Pearce, S. K., Herald Bldg., Calgary

Peardon, Thos. P., Columbia University, New York, N.Y.

Pearson, John A., Architect, Toronto

Pearson, L. B., Dept. of External Affairs, Ottawa

Pelletier, Dr. Arthur, 111 rue Notre-Dame, Joliette, P.Q.

Perley, Hon. Sir George H., 21 Blackburn Ave., Ottawa

Peterborough Public Library, Peterborough, Ont.

Pickersgill, J. W., Wesley College, Winnipeg

Pierce, Donald J., 537 Jarvis St., Toronto

Pierce, Dr. Lorne, The Ryerson Press, Toronto

Pitfield, W. C., "Saraguay", Cartierville, P.Q.

Planta, Mrs. Albert E., 325 Newcastle Ave., Nanaimo, B.C.

Plaunt, Alan B., University Club of Toronto, Toronto

Ponton, Lieut.-Col. W. N., Bridge St., Belleville, Ont.

Pritchett, John Perry, University of North Dakota, Grand Forks, N.D.

Public Archives of Canada, Ottawa

Québec, Département de l'Instruction Publique, Québec

Québec, Ministre des Terres et Forêts, Québec

Raymond, Raoul, 2402 St. Catherine St. E., Montreal

Reford, R. W., 20 Hospital St., Montreal

Reid, R. L., K.C., Yorkshire Bldg., Vancouver

Riddell, Hon. W. R., Osgoode Hall, Toronto

Rife, C. W., Hamline University, St. Paul, Minn.

Rimouski, Evêque de Rimouski, Que.

Robertson, Miss Margaret A., Public Archives of Canada, Ottawa

Robinson, W. Beverley, 906 McGill Building, Montreal

Roper, Right Rev. J. C., 140 Bay St., Ottawa

Ross, Mgr. F. X., Evêque de Gaspé, Gaspé, P.Q.

Ross, John T., 110 St. Peter St., Quebec

Rowell, Hon. N. W., 134 Crescent Road, Toronto

Roy, M. l'abbé Camille, Université Laval, Québec
Roy, Ferdinand, Palais de Justice, Québec Roy, Pierre-Georges, 44 Wolfe St., Lévis, P.Q.

Royal Institute of International Affairs, St. James Square, London, S.W. 1, England

Rutherford, Hon. A. C., 916 McLeod Building, Edmonton

Sage, W. N., University of British Columbia, Vancouver

Ste-Anne de la Pocatière, Collège de Ste. Anne de la Pocatière, P.Q.

St-Hyacinthe, Séminaire de, St-Hyacinthe,

P.Q.
St-Joseph Université, H. A. Vanier, C.S.C., St-Joseph, N.B.

Ste-Thérèse, Corp. Séminaire, Donat Godin, ptre, Proc., Ste-Thérèse, P.Q.

Savelle, Max, Barnard College, Columbia University, N.Y.

Scott, Duncan C., Dept. of Indian Affairs, Ottawa

Scott, S. Morley, 518 Linden St., Ann Arbor, Mich.

Scott, Dr. W. H., 416 McLeod Building, Edmonton

Sherwood, Sir Percy, 451 Daly Avenue, Ottawa

Shiels, Archie W., Pacific American Fisheries, South Bellingham, Wash.

Shortt, Dr. Geo. E., 63 Cartier St., Ottawa

Sirois, Joseph, rues Couillard et Christie, Québec

Sissons, C. B., Victoria College, Toronto

Sjostedt, Mrs. Jessie Winslow, 237 Chapel St., Ottawa

Skelton, Dr. O. D., Dept. of External Affairs, Ottawa

Smith, Geo. M., Dept. of History, University of Alberta, Edmonton

Smith, Joe Patterson, Illinois College, Jacksonville, Ill.

Smith, Mrs. Leslie V., 61 Lytton Blvd., Toronto

Somerville, C. R., 16 Kensington Apts., London, Ont.

Somerville, Mrs. J. M., Kenniston Apts., Ottawa

Southam Publishing Co., 1070 Bleury St., Montreal

Soward, F. H., Dept. of History, University of British Columbia, Vancouver

Spry, Graham, The Farmer's Sun, Toronto

Stacey, C. P., Graduate College, Princeton, N.J.

Starnes, Major-General Cortlandt, St. Hilaire, Co. Rouville, P.Q.

Staton, Miss Frances, Cawthra Mansions, Apt. 138, 211 College St., Toronto

Stevens, Hon. H. H., Parliament Buildings, Ottawa

Story, Miss Norah, Public Archives, Ottawa 


\section{(C) ANNUAL MEMBERS-Continued}

Stuart, Sir Campbell, The Times, Printing House Square, London, E.C. 4, England

Sullivan, A., Dept. of Education, Victoria

Sutherland, J. C., Dept. of Education, Quebec

Swannell, Frank, 564 Dallas Rd., Victoria

Talman, James J., 88 Delaware Ave., Toronto

Taschereau, Hon. L. A., Hôtel du Gouvernement, Québec.

Thomson, G. C., Barrister-at-law, Swift Current, Sask.

Thomson, R. B., Botany Building, University of Toronto, Toronto

Tilley, Leonard T. D., K.C., 42 Princess St., Saint John, N.B.

Tombs, Guy, 1111 Beaver Hall Hill, Montreal

Tombs, L. C., 503 Mount Pleasant Ave., Westmount, P.Q.

Trigge, A. St, L., P.O. Box 121, Melbourne, P.Q.

Trois-Rivières, Séminaire des, TroisRivières, P.Q.

Trois-Rivières, Société d'Histoire Régionale de, Séminaire des Trois-Rivières, P.Q.

Trotter, Reginald G., Queen's University, Kingston

Tucker, Gilbert Norman, The Deanery, London, Ont.

Tyrrell, J. B., 14 Walmer Rd., Toronto

Underhill, Frank H., Baldwin House, University of Toronto, Toronto

Vinet, Rev. Jean Baptiste, Collège de Montréal, 1931 rue Sherbrooke-ouest, Montréal

Wallace, Malcolm W., University College, University of Toronto, Toronto
Walter, T. Dayman, 1513 Church St., Windsor, Ont.

Ward, Miss Clare, 187 Indian Road, Toronto

Warner, Clarence, 50 Congress St., Boston, Mass.

Watson, S. B., 27 McLennan St., Toronto

Webster, Dr. J. C., Shediac, N.B.

Whitelaw, W. M., McGill University, Montreal

Williams, David, Collingwood, Ont.

Williams, Fred G. H., 310 Roncesvalles Ave., Toronto

Williams-Taylor, Sir Frederic, Kinnaird House, 1 Pall Mall, London, S.W. 1, England

Wills, Harold A., 10 Montague St., Toronto

Wilson, Clifford P., 52 Summit Circle, Westmount, P.Q.

Wilson, George E., Dalhousie University, Halifax

Winslow, Edward Pelham, 8 Trafalgar Place, Montreal

Wittke, Carl F., Ohio State University, Columbus, Ohio

Wood, Col. Wm., 59 Grande-Allée, Quebec

Woodyatt, James B., 5 Hudson Ave., Westmount, P.Q.

Wrong, G. M., 73 Walmer Rd., Toronto Wrong, H. H., Canadian Legation, Washington, D.C.

Wurtele, Lieut.-Col. E. F., Box 67, Station B, St. Catherine St. W., Montreal

Young, Dr. Archibald Hope, Trinity College, Toronto

Young, Norman, Ravenscourt School, Armstrong Point, Winnipeg 